K. ATSUYAMA

KODAI MATH. J

3 (1980), 358-363

\title{
SYMMETRIC SPACES DERIVED FROM ALGEBRAS
}

\author{
By Kenji AtSUYAMA
}

\section{$\S 1$. Introduction.}

The real projective plane is simply realized as the set of all lines through the origin in the 3-dimensional Euclidean space. And also it can be realized as the set of all subalgebras which are isomorphic to the field of complex numbers in the quaternion field. However there is a slight difference between two realizations, that is, in the former case the lines appear to have no algebraic structure but in the latter case the subalgebras do have it, by which the same symmetric space can be obtained. Then, since it seems to us that the similar realization to the latter is suitable for the explicit construction of symmetric spaces from various algebras, we will ask whether symmetric spaces in the sense of O. Loos [3] can be constructed by the set of all subalgebras with suitable conditions in a given algebra. In this paper we shall give an affirmative answer to this problem.

\section{$\S 2$. Preliminaries.}

Let $K$ be the field of real numbers (or the field of complex numbers) and $K^{n}$ be the $n$-dimensional vector space over $K$. We assume that $K^{n}$ has a non-trivial product $\mu$, i. e., a $K$-bilinear mapping $\mu: K^{n} \times K^{n} \rightarrow K^{n}$ such that $A \cdot A \neq\{0\}$ where we put $x \cdot y=\mu(x, y)$ and $A=\left(K^{n}, \mu\right) . A$ is a non-associative algebra. Then the general linear group $G L(n, K)$ of $K^{n}$ is a Lie group and the automorphism group Aut $(A)$ of $A$, the group of all elements $\alpha$ of $G L(n, K)$ which satisfy $\mu(\alpha x, \alpha y)$ $=\alpha \mu(x, y)$ for any $x, y \in A$, is also a Lie group because Aut $(A)$ is a closed subgroup in $G L(n, K)$. Moreover we assume that $A$ has a non-degenerate symmetric bilinear mapping (=inner product) $g: A \times A \rightarrow K$ which is invariant under $\operatorname{Aut}(A)$. Throughout this paper the product $\mu$ and the inner product $g$ will be fixed.

A subspace $V$ of the algebra $A$ is regular if $A$ is a direct sum of $V$ and $V^{\perp}$ $\left(A=V \oplus V^{\perp}\right)$ as a vector space where $V^{\perp}$ is a subspace of all elements $x$ of $A$ which are orthogonal to $V$ relative to $g$, i. e., $g(x, V)=\{0\}$. Since the inner product $g$ is symmetric and non-degenerate, for a regular subspace $V$, we can obtain a basis $\left\{e_{i}\right\}$ in $A$ satisfying the conditions $(*): e_{\imath} \in V\left(1 \leqq_{\imath} \leqq r\right), e_{i} \in V^{\perp}(r+1 \leqq \imath \leqq n)$

Received June 18, 1979

This research was partially supported by the Scientific Research Grant of the Ministry of Education, 1978-D-364035. 
and $g\left(e_{\imath}, e_{j}\right)=0$ or non-zero according as $\imath \neq \jmath$ or $\imath=\jmath$ where $r$ is the dimension of $V(\operatorname{dim} V)$ and $n=\operatorname{dim} A$.

A symmetric subalgebra $V$ is a regular subspace of $A$ such that $V \cdot V \subset V$, $V \cdot V^{\perp} \subset V^{\perp}, V^{\perp} \cdot V \subset V^{\perp}$ and $V^{\perp} \cdot V^{\perp} \subset V$. By the assumption of $A \cdot A \neq\{0\}$, the dimension of a symmetric subalgebra is always positive. These symmetric subalgebras play an important role in the explicit construction of symmetric spaces from various algebras.

\section{§3. Reflection map $S_{V}$.}

We define a reflection map $S_{V}$ in the algebra $A$ across a regular subspace $V$ by $S_{V}\left(x_{1}+x_{2}\right)=x_{1}-x_{2}$ for any $x_{1} \in V$ and $x_{2} \in V^{\perp}$ and also define a reflection map $S_{a}$ in $A$ across a non-isotropic vector $a$, i. e., $g(a, a) \neq 0$, by $S_{a}(x)=-x+2 g(x, a) /$ $g(a, a) a$ for any $x \in A$. Then the determinant $\operatorname{det} S_{a}$ is equal to $(-1)^{n-1}$ where $n=\operatorname{dim} A$. If $g(x, a)=0, S_{a}(x)=-x$.

Let $\varepsilon$ be -1 or 1 according as $\operatorname{dim} V$ is even or odd. For a regular subspace $V$ of $A$, making use of a basis $\left\{e_{i}\right\}$ of $A$ with the property (*) in Section 2, we can obtain $S_{V}=\varepsilon \Pi S_{e_{i}}$ where $\Pi$ is the composition of $r$ reflection maps.

Proposition 3.1. Let $V$ be a regular subspace of $A$, then the reflection map $S_{V}$ is an (involutive) automorphism of $A$ if and only if $V$ is a symmetric subalgebra.

Proof. Sufficiency: From det $S_{V} \neq 0, S_{V} \in G L(n, K)$. Since, for $x, y \in V$ and $z, w \in V^{\perp}$, it holds that $S_{V}(x \cdot y)=x \cdot y=S_{V}(x) \cdot S_{V}(y), S_{V}(x \cdot z)=-x \cdot z=S_{V}(x) \cdot S_{V}(z)$, $S_{V}(z \cdot x)=-z \cdot x=S_{V}(z) \cdot S_{V}(x)$ and $S_{V}(z \cdot w)=z \cdot w=S_{V}(z) \cdot S_{V}(w), S_{V}$ is an automorphism of $A$.

Necessity: Notice that $V=\left\{x \in A \mid S_{V}(x)=x\right\}$ and $V^{\perp}=\left\{z \in A \mid S_{V}(z)=-z\right\}$. Since we have $S_{V}(x \cdot y)=S_{V}(x) \cdot S_{V}(y)=x \cdot y, S_{V}(x \cdot z)=S_{V}(x) \cdot S_{V}(z)=-x \cdot z, S_{V}(z \cdot x)$ $=S_{V}(z) \cdot S_{V}(x)=-z \cdot x$ and $S_{V}(z \cdot w)=S_{V}(z) \cdot S_{V}(w)=z \cdot w$ for any $x, y \in V$ and $z, w$ $\in V^{\perp}, V$ is a symmetric subalgebra.

Lemma 3.2. For a regular subspace $V$ of $A, \alpha S_{V} \alpha^{-1}=S_{\alpha V}$ holds for any $\alpha \in$ $\operatorname{Aut}(A)$.

Proof. For a non-isotropic vector $a$ of $A$, we have $\alpha S_{a} \alpha^{-1}(x)=$ $\alpha\left(-\alpha^{-1} x+2 g\left(\alpha^{-1} x, a\right) / g(a, a) a\right)=-x+2 g(x, \alpha a) / g(\alpha a, \alpha a) \alpha a=S_{\alpha a}(x)$ for any $\alpha \in$ Aut $(A)$ and $x \in A$, i. e., $\alpha S_{a} \alpha^{-1}=S_{\alpha a}$ holds. From this fact and $S_{V}=\varepsilon \Pi S_{e_{i}}$, we can prove the Lemma.

The following Proposition is a direct consequence of Lemma 3.2.

Proposition 3.3. Let $V$ be a symmetric subalgebra of $A$. Then the group generated by the set $\mathfrak{S}_{V}$ of all $S_{\alpha V}$ for any $\alpha \in$ Aut $(A)$ is a normal subgroup of Aut $(A)$. If Aut $(A)$ is simple (in the sense of abstract group) and $V \neq A, \mathfrak{S}_{V}$ generates $\operatorname{Aut}(A)$. 
Proposition 3.4. If $\alpha$ is an involutive automorphism of $A$, there exists uniquely a symmetric subalgebra $V$ such that $\alpha=S_{V}$.

Proof. Since the involutive automorphism $\alpha$ can be represented as an $n \times n$ matrix with coefficients in $K$, there is an $n \times n$ non-singular matrix $P$, i. e., $P \in$ $G L(n, K)$, such that $\alpha=P D P^{-1}$ where $D$ is a diagonal matrix whose diagonal components are \pm 1 . Let $W_{ \pm 1}$ be \pm 1 -eigenspaces for this diagonal matrix $D$, then we have $A=W_{1} \oplus W_{-1}$ (direct sum as a vector space). And, put $P W_{1}=V_{1}$ and $P W_{-1}=V_{-1}$, we also have $A=V_{1} \oplus V_{-1}$ such that $V_{1}=\{x \in A \mid \alpha(x)=x\}$ and $V_{-1}=\{x \in A \mid \alpha(x)=-x\}$. Since the involutive automorphism $\alpha$ can be characterized by these vector spaces, it suffices to prove that $V_{1}$ is a symmetric subalgebra such that $\alpha=S_{V_{1}}$. If $V_{1}=\{0\}$, it holds that $y \cdot z=\alpha(y) \cdot \alpha(z)=\alpha(y \cdot z)=-y \cdot z$ for any $y, z \in V_{-1}(=A)$, i. e., $A \cdot A=\{0\}$. However this contradicts the assumption of $A \cdot A \neq\{0\}$ and so the case of $V_{1}=\{0\}$ does not occur. If $V_{-1}=\{0\}$ we can take $A$ for $V$ such that $\alpha=S_{V}$. Hence we only consider a case of $V_{ \pm 1} \neq\{0\}$.

First we show $V_{-1}=V_{1}^{\perp}$. Since the inner product $g$ is invariant under the automorphism group Aut $(A)$, we have $g(x, y)=g(\alpha x, \alpha y)=g(x,-y)=-g(x, y)$, i. e., $g(x, y)=0$ for any $x \in V_{1}, y \in V_{-1}$. This implies $V_{-1} \subset V_{1}^{\perp}$. From $A=V_{1} \oplus V_{-1}$ and the fact that $\operatorname{dim} V^{\perp}=n-\operatorname{dim} V$ holds in general under the assumption of the existence of a non-degenerate symmetric inner product, we have $V_{-1}=V_{1}^{\perp}$. This means that $V_{1}$ is a regular subspace of $A$. Next, from the fact that $\alpha(x \cdot y)$ $=\alpha(x) \cdot \alpha(y)=x \cdot y, \alpha(x \cdot z)=\alpha(x) \cdot \alpha(z)=-x \cdot z, \alpha(z \cdot x)=\alpha(z) \cdot \alpha(x)=-z \cdot x$ and $\alpha(z \cdot w)$ $=\alpha(z) \cdot \alpha(w)=z \cdot w$ for any $x, y \in V_{1}$ and $z, w \in V_{1}^{1}$, we can see that $V_{1}$ is a symmetric subalgebra of $A$ such that $\alpha=S_{V_{1}}$.

Two involutive automorphisms $\alpha, \beta$ are conjugate if there exists a $\gamma \in \operatorname{Aut}(A)$ such that $\alpha=\gamma \beta \gamma^{-1}$. Two symmetric subalgebras $V, W$ are conjugate if there exists a $\gamma \in \operatorname{Aut}(A)$ such that $V=\gamma W$. Since $S_{V}=S_{W}$ is equivalent to $V=W$, by Propositions 3.1, 3.4 and Lemma 3.2, we have

Proposition 3.5. The conjugate classes of involutive automorphisms of $\operatorname{Aut}(A)$ are in one-to-one correspondence with the conjugate classes of symmetric subalgebras of $A$.

\section{$\S 4$. Main result.}

Let $V$ be a symmetric subalgebra of $A$. We define a multiplication $\circ$ on $\Im_{V}$ by $\alpha \circ \beta=\alpha \beta \alpha$ where the set $\widetilde{S}_{V}$ is the conjugate class of $S_{V}$ in Aut $(A)$. Then we can easily obtain that 1) $\alpha \circ \alpha=\alpha, \alpha \circ(\alpha \circ \beta)=\beta$ and $\alpha \circ(\beta \circ \gamma)=(\alpha \circ \beta) \circ(\alpha \circ \gamma)$ for any $\alpha, \beta, \gamma \in \mathbb{S}_{V}$. Under the transformation map $\psi: \operatorname{Aut}(A) \times \operatorname{Aut}(A) \rightarrow \operatorname{Aut}(A)$ : $(u, v) \rightarrow u v u^{-1}$, if we fix $v=S_{V}$, the set $\Im_{V}$ becomes an orbit on which an analytic structure can be uniquely introduced such that 2) $\mathfrak{S}_{V}$ is diffeomorphic to Aut $(A) / H$ where $H$ is the isotropic subgroup of $v=S_{V}$ relative to $\left.\phi\left(\Im_{V} \cong \operatorname{Aut}(A) / H\right), 3\right) \widetilde{\varsigma}_{V}$ is a submanifold of $\operatorname{Aut}(A)$ (if $\operatorname{Aut}(A)$ is compact, it is a regular submanifold), 4) the multiplication $\circ$ on $\mathfrak{S}_{V}$ is analytic, 5) Aut $(A)$ is the Lie transformation 
group on $\left.\mathfrak{S}_{V}, 6\right)$ for every $u \in \operatorname{Aut}(A)$, the map $\tau(u): \widetilde{S}_{V} \rightarrow \Im_{V}: S_{\alpha V} \rightarrow S_{u \alpha V}$ is a diffeomorphism and it is also a homomorphism relative to the multiplication $\circ$ on $\Im_{V}$ (cf. S. Helgason, p. 113 [2]) and at last 7) the following Lemma holds.

Lemma 4.1. For any $\alpha \in \Im_{V}$, there exists a neighborhood $U$ of $\alpha$ in $\Im_{V}$ such that $\alpha \circ \beta=\beta, \beta \in U$ implies $\alpha=\beta$.

Proof. From the property 6) above, it is sufficient to prove only in the case of $\alpha=S_{V}$. Suppose this Lemma is not true, then we have a countable sequence $\left\{\beta_{l}\right\}$ in $\widetilde{S}_{V}$ such that $\alpha \circ \beta_{l}=\beta_{l}$ with $\beta_{l} \neq \alpha$ for every $l \in N$ and $\lim \beta_{l}=\alpha$. Since we can find a local section $h: U \subset \mathfrak{S}_{V} \rightarrow \operatorname{Aut}(A)$ such that $h(\alpha)=1$ and $\pi h$ is the identity map on the neighborhood $U$ of $\alpha$ where 1 is the identity element of Aut $(A)$ and $\pi$ is the projection map from $\operatorname{Aut}(A)$ onto $\widetilde{S}_{V}\left(\operatorname{Aut}(A) / H \cong \Im_{V}\right.$ ), we also have a sequence $\left\{\gamma_{l}\right\}$ in Aut $(A)$ such that $\beta_{l}=\gamma_{l} \alpha \gamma_{l}^{-1}$ with $\gamma_{l} \neq 1$ for any $l \in N$ and $\lim \gamma_{l}=1$. By Proposition 3.4, moreover, we can take a set $\left\{W_{l}\right\}$ of symmetric subalgebras such that $S_{W_{l}}=\beta_{l}$ for every $l \in N$. Then it holds $W_{l}=\gamma_{l} V$ and $\alpha W_{l}$ $=W_{l}$ because $S_{Z}=S_{W}$ is equivalent to $Z=W$ and $\alpha \circ \beta_{l}=\beta_{l}$.

Now we take a basis $\left\{e_{\imath}\right\}$ of $A$ for the symmetric subalgebra $V$ with the property (*) in Section 2. Put $\Delta_{l, i}=\gamma_{l} e_{i}-e_{\imath}$, then $\lim \Delta_{l, \imath}=0$ because $\lim \gamma_{l}=1$. Notice that $\left\{\gamma_{l} e_{m}\right\}, 1 \leqq m \leqq r$ and $r=\operatorname{dim} V$, is a basis for $W_{l}$. Next, making use of $S_{V}=\varepsilon \Pi S_{e_{m}}$, we have $S_{V}\left(\gamma_{l} e_{m}\right)+\gamma_{l} e_{m}=S_{V}\left(e_{m}+\Delta_{l, m}\right)+\gamma_{l} e_{m}=2 e_{m}+2 \Sigma g\left(\Delta_{l, m}, e_{k}\right) /$ $g\left(e_{k}, e_{k}\right) e_{k}$ (put this element as $\left.2 w_{l, m}\right)$. Since $S_{V}\left(W_{l}\right)=W_{l}, w_{l, m} \in W_{l}$ holds. Put $a_{l, \imath j}=g\left(\Delta_{l, \jmath}, e_{\imath}\right) / g\left(e_{\imath}, e_{\imath}\right)$ and $C_{l}=I+\left(a_{l, \imath \jmath}\right)$ for $1 \leqq i, \jmath \leqq r$, we obtain $\lim C_{l}=I$ by $\lim \Delta_{l, j}=0$ where $I$ is the $r \times r$ unit matrix. Hence there exists a positive integer $l_{0}$ such that $\operatorname{det} C_{l} \neq 0$ for $l \geqq l_{0}$. Put $C_{l}=\left(c_{l, \imath j}\right)$ and $C_{l}^{-1}=\left(d_{l, \imath j}\right)$ for $l \geqq l_{0}$. Then, from $\Sigma c_{l, i k} d_{l, k j}=\delta_{i j}$ and $\Sigma c_{l, k m} e_{k}=w_{l, m}$, we get $e_{\imath}=\Sigma d_{l, k i} w_{l, k} \in W_{l}(1 \leqq i \leqq r)$, i. e., $V=W_{l}$ because $\operatorname{dim} V=\operatorname{dim} W_{l}$. However this contradicts $V \neq W_{l}$.

The properties of 1 ), 4) and Lemma 4.1 assert that the orbit $\mathfrak{S}_{V}$ is a symmetric space in the sense of $\mathrm{O}$. Loos [3]. In conclusion we have a following main result.

THEOREM 4.2. Let $A$ be a non-associative algebra over the field of real numbers (or complex numbers) having a non-trivial product and a non-degenerate symmetric inner product which is invariant under Aut $(A)$. Let $V$ be a proper symmetric subalgebra of $A(V \neq A)$, if we put $\mathfrak{S}_{V}=\left\{S_{\alpha V} \mid \alpha \in \operatorname{Aut}(A)\right\}$, then we have

1) $\mathfrak{S}_{V}$ is a symmetric space,

2) $\mathfrak{S}_{V}$ is a submanifold of Aut $(A)$. If Aut $(A)$ is compact, $\mathfrak{S}_{V}$ is a regular submanifold,

3) If Aut $(A)$ is a simple group, $\widetilde{S}_{V}$ generates Aut $(A)$,

4) Every element of $\widetilde{S}_{V}$ is a non-trivial involutive automorphism,

5) Aut $(A)$ is the Lie transformation group on $\mathfrak{S}_{V}$,

6) For every $u \in$ Aut $(A)$, the map $\tau(u)$ of $\widetilde{S}_{V}: S_{\alpha V} \rightarrow S_{u \alpha V}$ is a diffeomorphrsm and moreover it is a homomorphism relative to the multiplication $\circ$ on $\widetilde{S}_{V}$,

7) If we define a new multiplication $u \circ v=u v^{-1} u$ in $\operatorname{Aut}(A)$, Aut $(A)$ also 
becomes a symmetruc space. Then the embedding of 2) above from $\mathfrak{S}_{V}$ into $\operatorname{Aut}(A)$ is a homomorphism of symmetruc spaces (see O. Loos [3] and K. Atsuyama [1]).

\section{$\S 5$. Examples.}

5.1. Let $\boldsymbol{Q}$ be the quaternion field with a basis $\left\{e_{i}\right\}(0 \leqq \imath \leqq 3)$ such that $e_{0}$ is the unit element $(=1), e_{1} e_{2}=e_{3}, e_{2} e_{3}= \pm e_{1}, e_{3} e_{1}=e_{2}, e_{i} e_{j}=-e_{\jmath} e_{2}$ for $\imath \neq j \geqq 1$ and $e_{1}{ }^{2}$ $=-1, e_{\imath}{ }^{2}=-1$ (or 1 ) for $\imath=2,3$ : in case of $e_{\imath}{ }^{2}=-1$ for every $i \geqq 1, \boldsymbol{Q}$ is called non-split and in the other case of $e_{1}{ }^{2}=-1, e_{2}{ }^{2}=1$ and $e_{3}{ }^{2}=1, \boldsymbol{Q}$ is called split. Then Aut $(\boldsymbol{Q})$ is the special orthogomal linear group $S O(3)$ or $S O(2,1)$ respectively and it is a simple group. The non-degenerate symmetric inner product $g$ is given by $g(x, x)=x \bar{x}$ and it is invariant under Aut $(\boldsymbol{Q})$. In case of non-split type there exists only one conjugate class $\widetilde{S}_{V}$ of which the symmetric subalgebra $V$ is generated by $e_{0}, e_{1}$, i. e., isomorphic to the field of complex numbers. Then the real projective plane can be obtained by $\widetilde{\varsigma}_{V}=S O(3) / S O(2) \cdot S^{0}$ where $\cdot$ is the semidirect product and $S^{0}$ is the group of \pm 1 . In case of split type there are two conjugate classes $\widetilde{S}_{Z}, \Im_{W}$ of which $Z$ is generated by $e_{0}, e_{1}$ and $W$ by $e_{0}, e_{2}$. Then $\widetilde{\subseteq}_{z}=S O(2,1) / S O(2) \cdot S^{0}$ is a real hyperbolic plane and $\Im_{W}=S O(2,1) / S O(1,1) \cdot S^{0}$ is a real parabolic plane. We can also assert that the automorphisms of the quaternion field are the reflection maps across symmetric subalgebras which are isomorphic to the field of complex numbers.

5.2. Let $\left[5\right.$ be the Cayley algebra with a basis $\left\{e_{i}\right\}(0 \leqq i \leqq 7)$ such that the subalgebra generated by $\left\{e_{i}\right\} \quad(0 \leqq \imath \leqq 3)$ is isomorphic to the quaternion field $\boldsymbol{Q}$ of non-split type (again denote this subalgebra by $\boldsymbol{Q}$ ), $e_{\imath}{ }^{2}=-1$ (or 1 ) for $4 \leqq \imath \leqq 7$. We call $(5)$ non-split or split respectively. Then Aut(ङ) is the exceptional simple Lie group of type $\boldsymbol{G}_{2}$. The non-degenerate symmetric inner product $g$ is given by $g(x, x)=x \bar{x}$ and it is invariant under Aut(ङ). If $(\xi)$ is non-split there is one class $\mathfrak{S}_{Q}$ diffeomorphic to Aut (⿷) $/ S O(4)=G_{2} / S O(4)$. If $(5$ is split there are two classes $\mathfrak{S}_{V}, \mathfrak{S}_{W}$ of which $V$ is isomorphic to the non-split quaternion field and $W$ to the split quaternion field. Then we have two symmetric spaces: $\varsigma_{V}=$ Aut (ङ) $/ S O(4)=\boldsymbol{G}_{2}{ }^{*} / S O(4)$ is a hyperbolic space and $\widetilde{S}_{W}=\boldsymbol{G}_{2}{ }^{*} / S O(2,1) \cdot H^{*}$ is a parabolic space where $*$ means the non-compact type of the corresponding group and $H^{*}$ is the group generated by elements $q$ of the split quaternion field with $g(q, q)=1$. $\quad \widetilde{S}_{Q}$ is an elliptic projective space. We can also assert that the automorphisms of the Cayley algebra are reflection maps across symmetric subalgebras which are isomorphic to the quaternion field and their forms are $\varepsilon S_{e_{0}} S_{a} S_{b} S_{a b}$ where $e_{0}, a, b$ and $a b$ are orthogonal each other.

5.3 Let $\Im$ be the exceptional Jordan algebra with the Jordan product $X \circ Y=$ $1 / 2(X Y+Y X)$ for $X, Y \in \Im$. It is generated by $3 \times 3$ Hermitian matrices with

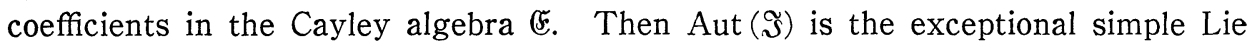
group of type $\boldsymbol{F}_{4}$. The non-degenerate symmetric inner product $g$ is given by $g(X, X)=\operatorname{Tr}\left(L_{X \circ X}\right)$ for $X \in \Im$ where $\operatorname{Tr}$ is the trace form and $L_{X} Y=X \circ Y$ for $Y \in \mathfrak{S}$ and it is invariant under Aut (予). If $(\xi$ is non-split there are two conjugate classes $\widetilde{\varsigma}_{V}, \widetilde{\varsigma}_{W}$ of which $V$ is generated by all matrices with coefficients in the 
quaternion field of non-split type and the other $W$ by all matrices $X=X\left(a_{\imath j}\right)$ with $a_{1 \imath}=0, \quad a_{i 1}=0$ for $\imath=2,3$. Then we have $\Im_{W}=\operatorname{Aut}(\Im) / \operatorname{Spin}(9)=\boldsymbol{F}_{4} / \operatorname{Spin}(9)$ and $\widetilde{S}_{V}=\boldsymbol{F}_{4} / T$ where $T=S p(3) \cdot H / S^{0}$ with $H$ being the group of all elements $q$ of the non-split quaternion field such that $g(q, q)=1$. Since, for every element of $\mathfrak{S}_{W}$, there corresponds uniquely one element of $\Im$ s, the Cayley plane $\boldsymbol{F}_{4} / \operatorname{Spin}(9)$ can be realized in $\mathfrak{s}$ as the set of all matrices $X=X\left(a_{\imath j}\right)$ with $X \circ X=X$ and $\Sigma a_{i i}=1$. If $\left(5\right.$ is split we obtain three conjugate classes $\mathfrak{S}_{V}, \Im_{W}, \Im_{Z}$ where $V$ is composed of all matrices $X=X\left(a_{\imath j}\right)$ with $a_{1 \imath}=0, a_{i 1}=0$ for $\imath=2,3$ and $W$ (or $Z$ ) of all matrices with coefficients in the non-split (or split) quaternion algebra: $\widetilde{S}_{V}=\operatorname{Aut}(\Im) / \operatorname{Spin} *(9)=\boldsymbol{F}_{4} * / \operatorname{Spin} *(9)$ and the latter two symmetric spaces correspond to $\boldsymbol{F}_{4} / T$, i. e., $\varsigma_{W}=\boldsymbol{F}_{4} * / T$ is a hyperbolic space and $\varsigma_{Z}=\boldsymbol{F}_{4} * / T^{*}$ is a parabolic space where $T^{*}=S p^{*}(3) \cdot H^{*} / S^{0}$ (see I. Yokota [6]).

5.4. For simple Lie algebras we can take the Killing form as a non-degenerate symmetric inner product. Especially it may be suitable to use the algebras obtained by the Tits' second construction for the realization of symmetric spaces from exceptional Lie algebras (see Tits [5]).

5.5. For algebras of $n \times n$ matrices there are two standard forms of symmetric subalgebras: the one is composed of matrices $X=X\left(a_{\imath j}\right)$ with $a_{\imath j}=0, a_{\imath j}=0$ for $1 \leqq \imath \leqq p, p+1 \leqq j \leqq n$ and fixed $p$ and the other of matrices with coefficients in the real part relative to the construction of complex numbers, quaternion numbers and Cayley numbers by the Cayley-Dickson process (cf. R. D. Schafer, p. 45 [4]).

\section{REFERENCES}

[1] K. Atsuyama, On the embedding of the Cayley plane into the exceptional Lie group of type $\mathbf{F}_{4}$, Kōdai Math. Sem. Rep. 28 (1977), 129-134.

[2] S. Helgason, Differential geometry and symmetric spaces, Academic Press, New York, 1962.

[3] O. Loos, Symmetric spaces I, Benjamin, New York, 1969.

[4] R.D. Schafer, An introduction to nonassociative algebras, Academic Press, New York, 1966.

[5] J. Tits, Algebres alternatives, algebres de Jordan et algebres de Lie exceptionnelles, I. Construction, Nederl. Akad. Weten. Proc. Ser. A, 69 (1966), 223-237.

[6] I. ҮокотA, Non-compact simple Lie group $\mathbf{F}_{4,2}$ of type $\mathbf{F}_{4}$, J. Fac. Scı. Shinshu Univ., 12 (1977), 53-64.

Kumamoto Institute of Technology

KumAMOTO, IKeda, 860, Japan. 